\title{
Mechanisms of oxygen inhibition of nirK expression in Rhodobacter sphaeroides
}

\begin{abstract}
Correspondence
James P. Shapleigh

jps2@cornell.edu
\end{abstract}

Received 2 February 2010

Revised 24 June 2010

Accepted 28 June 2010

\author{
Angela Hartsock and James P. Shapleigh
}

Department of Microbiology, Wing Hall, Cornell University, Ithaca, NY 14853, USA

\section{INTRODUCTION}

Denitrification is the dissimilatory reduction of nitrogen oxyanions to gaseous end products (Zumft, 1997). This process is secondary to aerobic respiration and is utilized as an alternative respiratory mode under micro-oxic and anoxic conditions. Denitrification is widespread in the biological world with eukaryotes, fungi, archaea and bacteria being able to respire fixed nitrogen oxyanions to gaseous forms (Risgaard-Petersen et al., 2006; Zumft, 1997). An obligate intermediate in the denitrification pathway is nitric oxide (NO), produced upon nitrite reduction. The production of $\mathrm{NO}$ is believed to act directly as a signal for transcriptional regulators of denitrification genes, including the genes encoding nitrite and NO reductase (Giardina et al., 2008; Kwiatkowski \& Shapleigh, 1996; Spiro, 2007; Tosques et al., 1996). NO is also a reactive free radical so its production must be tightly controlled. Consistent with this tight control, studies of expression of nirK, the gene encoding a copper-containing nitrite reductase, in some denitrifiers has revealed that its expression requires multiple regulators that integrate information about levels of oxygen and nitrogen oxides (Baek et al., 2008; Bedmar et al., 2005; de Bruijn et al., 2006; Laratta et al., 2002). For example, in Agrobacterium tumefaciens the nirK gene has two levels of regulation. nirK is regulated first by ActR, a global transcriptional regulator, and second by $\mathrm{NnrR}$, a denitrification specific regulator (Baek et al., 2008).

Rhodobacter sphaeroides strain 2.4.3 is a model for denitrification in an anoxygenic photoheterotroph. Strain 2.4.3 has a nirK and a cytochrome $c$-oxidizing nitric oxide reductase (Bartnikas et al., 1997; Tosques et al., 1997). Previously, the NnrR in this bacterium was established as an NO-responsive Fnr/Crp-type transcriptional regulator that regulates several genes involved in denitrification, including nirK (Hartsock \& Shapleigh, 2010; Kwiatkowski \& Shapleigh, 1996; Tosques et al., 1996). In addition to $\mathrm{NnrR}$, the global two-component regulatory system PrrAB is required for upregulation of nirK since inactivation of the genes encoding these proteins drastically reduces nirK expression (Laratta et al., 2002). PrrB is a membranebound histidine sensor kinase and PrrA is the response regulator. As electron flow through the aerobic respiratory chain decreases, concomitant with oxygen levels, the kinase activity of PrrB is upregulated. In turn, PrrA is phosphorylated and activates or represses hundreds of genes that are involved in the transition from aerobic to anaerobic growth (Eraso et al., 2008). Importantly, the $c b b_{3}$ oxidase is required for inhibiting the kinase activity of PrrB (Oh et al., 2004). In the absence of a functional $c b b_{3}$ oxidase, PrrB constitutively phosphorylates PrrA, resulting in significantly higher levels of phosphorylated PrrA under aerobic conditions (Ranson-Olson \& Zeilstra-Ryalls, 2008). This uncouples the regulatory system from oxygen levels and allows PrrA-mediated activation or repression of genes under conditions of high $\mathrm{O}_{2}$ (O'Gara et al., 1998).

Contrary to the expectations based on the model of PrrAB function, it has been observed that nirK is not expressed in the absence of the $c b b_{3}$ oxidase (Laratta et al., 2002). Since PrrA-P levels are high in this background it would be expected that nirK and nor expression would not be impacted by the loss of the oxidase. Here, experiments 
undertaken to gain insight into the lack of nirK expression in the absence of the $c b b_{3}$ oxidase are described, and a model of why nirK is not expressed is presented.

\section{METHODS}

Bacterial strains and culture conditions. Two strains of Rhodobacter sphaeroides were used in this study. Strain 2.4.3 (ATCC 17025) was used as the model denitrifying strain and the type strain 2.4.1 (ATCC 17023) was used as a non-denitrifying comparison. Mutants of the strains used in this study are listed in Table 1 . The strains were cultured in Sistrom's medium at $32{ }^{\circ} \mathrm{C}$. When necessary, medium was supplemented with $12 \mathrm{mM}$ potassium nitrate to support denitrifying growth (Lueking et al., 1978). The flask culture conditions used have been previously described (Tosques et al., 1996). Briefly, $100 \mathrm{ml}$ liquid medium was added to $250 \mathrm{ml}$ Erlenmeyer flasks, which were then inoculated and sealed with a rubber stopper. Flasks were incubated at $32{ }^{\circ} \mathrm{C}$ and agitated at 250 r.p.m. Aerobic growth results in depletion of oxygen in the headspace and, for this ratio of head space to liquid, low-oxygen and denitrifying growth commences at optical densities above 0.6. Relevant antibiotics were added at the following concentrations: tetracycline, $1.0 \mu \mathrm{g} \mathrm{ml}^{-1}$; kanamycin, $25 \mu \mathrm{g} \mathrm{m}{ }^{-1}$; streptomycin, $50 \mu \mathrm{g} \mathrm{ml}^{-1}$; and gentamicin, $20 \mu \mathrm{g} \mathrm{ml}^{-1}$.

Escherichia coli DH5 $\alpha$ was used for molecular cloning and transformations. E. coli S17-1 was used for biparental matings. All E. coli cultures were grown in Luria-Bertani medium and liquid cultures were grown at $32{ }^{\circ} \mathrm{C}$ with aeration (Maniatis et al., 1982). When necessary, antibiotics were added at the following concentrations: ampicillin, $100 \mu \mathrm{g} \mathrm{ml}^{-1}$; tetracycline, $10 \mu \mathrm{g} \mathrm{ml}^{-1}$; streptomycin, $25 \mu \mathrm{g} \mathrm{ml}^{-1}$; and kanamycin, $25 \mu \mathrm{g} \mathrm{ml}^{-1}$.

Construction of plasmids and strains. The strains and plasmids used in this study are listed in Table 1. For PCRs, chromosomal DNA was isolated from $R$. sphaeroides strain 2.4.3 using the Puregene DNA Isolation kit. All oligonucleotide primers were purchased from Integrated DNA Technologies (IDT). Standard methods were used for restriction digests, ligations and biparental conjugations. Transformations were done using the TSS chemical method (Chung et al., 1989).

$\triangle \mathbf{c c o N}$ mutant construct. A $c b b_{3}$ oxidase mutant was made by deleting $c c o N$, which encodes the catalytic subunit. This was achieved by double recombination, resulting in the insertion of a streptomycinresistance cassette. To allow for a double-crossover event, two flanking regions of $c c o N$ were amplified and the amplicons were cloned together into pUC19, using either PstI/BamHI or BamHI/EcoRI. A streptomycinresistance cassette was inserted into the $\mathrm{BamHI}$ site; this process utilized

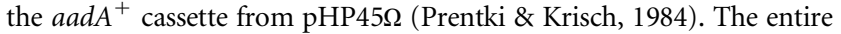
construct was then cloned into the EcoRI and PstI sites of the suicide vector pSUP202-1 (Simon et al., 1983). The construct was transformed into E. coli S17-1 and conjugated into R. sphaeroides 2.4.3. Transconjugants were selected on streptomycin medium and subsequently screened to confirm tetracycline sensitivity. The insertion in $c c o N$ was then verified by PCR.

nirK overexpression construct. For overexpression of nirK, an existing overexpression construct (pWLNIR) fusing prrnB' and nirK was moved into a new vector background, pBBR1-MCS5, by using HindIII and EcoRI. This new overexpression vector was termed pAKHNIR.

Enzyme assays. Relative gene expression levels for lac $Z$ fusions were measured by determining $\beta$-galactosidase activity (Maniatis et al., 1982). Activity was determined based on three independent cultures, and the mean values were calculated. Nir activity was determined using a colorimetric Nir assay (Laratta et al., 2002). Activity was determined for at least three independently grown cultures, and the mean values were calculated. Error bars represent one standard deviation in the figures. Oxygen uptake was measured using a Clarktype oxygen electrode.

Special culture conditions. For denitrifying growth at high r.p.m., cells were grown in sealed flasks as previously described (Tosques et al., 1996, 1997) at 250 r.p.m. For denitrifying growth at low r.p.m., flasks were grown in the dark on a bench-top rotary shaker at 50 r.p.m. For photo-denitrifying growth at high and low r.p.m., flasks

Table 1. Bacterial stains and plasmids used in this study

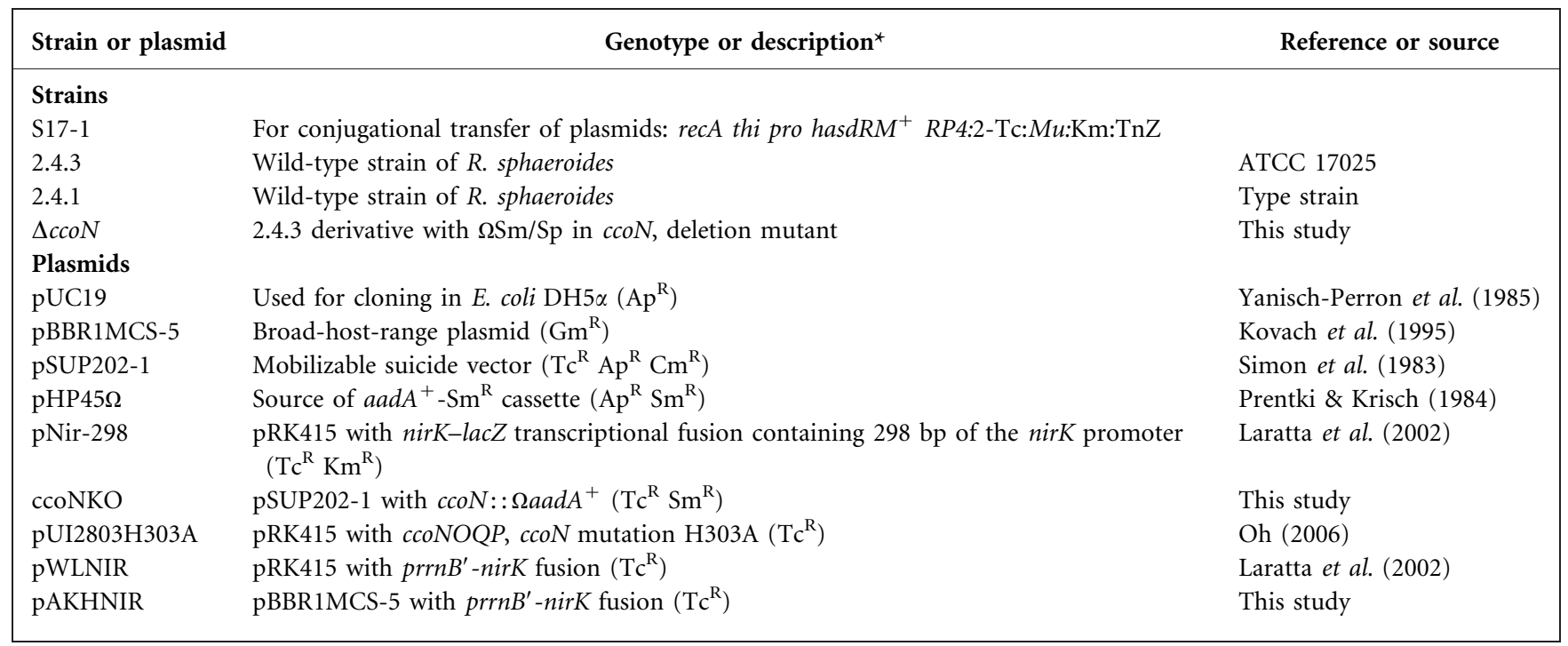

${ }^{\star}$ Tc, tetracycline; Km, kanamycin; Sm, streptomycin; Ap, ampicillin; Gm, gentamicin; Cm, chloramphenicol. 
were grown next to an incandescent light source at the r.p.m. mentioned above. For micro-oxic growth, plates were incubated in a polycarbonate anaerobic jar with the atmosphere three times evacuated and replaced with high-purity (minimum $99.995 \%$ ) $\mathrm{N}_{2}$ from Airgas.

Oxygen dilution experiments. Cells were grown in $125 \mathrm{ml}$ serum vials on solid slants of Sistrom's medium. Solid medium slants were made using $15 \mathrm{ml}$ culture medium. After inoculation the vials were sealed and evacuated for $40 \mathrm{~s}$. The vials were then flushed with $\mathrm{N}_{2}$ gas for $45 \mathrm{~s}$. Vials were equilibrated to atmospheric pressure using a water-filled syringe to allow trapped gas to escape without allowing atmospheric exchange. Leaving the gas-escape syringe in place, the desired amount of air was added back using a gas-tight syringe. Finally the gas-escape syringe was removed and the vials were incubated at $32{ }^{\circ} \mathrm{C}$ in the dark. For each percentage of oxygen in the headspace the following volumes of air were added back: $2 \%$, $11.1 \mathrm{ml} ; 1.3 \%, 7.4 \mathrm{ml} ; 0.67 \%, 3.7 \mathrm{ml} ; 0.45 \%, 2.5 \mathrm{ml} ; 0.22 \%, 1.2 \mathrm{ml}$; $0.13 \%, 0.73 \mathrm{ml} ; 0.07 \%, 0.36 \mathrm{ml}$ and $<0.07 \%$, no addition. The 'no addition' condition was also the micro-oxic condition used, since the $\mathrm{N}_{2}$ gas used contained enough oxygen to allow growth under dark conditions on medium lacking nitrate. The atmospheric control ( $20 \%$ oxygen) was evacuated and then allowed to equilibrate with atmospheric air using an open syringe.

\section{RESULTS}

\section{nirk expression in a $\mathrm{cbb}_{3}$ oxidase mutant}

The expression of nirK in a ccoN mutant background was previously studied using a strain in which $c c o N$ was inactivated via Campbell insertion of a suicide plasmid (Laratta et al., 2002). More recent work found that this insertion was unstable following prolonged incubation under micro-oxic conditions, allowing the restoration of $c b b_{3}$ activity. Therefore, a second $c c o N$ mutant was made in which 700 bases of $c c o N$ were deleted to eliminate the possibility of the restoration of $c b b_{3}$ activity. This strain was designated $\Delta c c o N$. As with the previous $c c o N$ mutant, $\Delta c c o N$ could not grow under micro-oxic conditions. Unlike the previous mutant, however, $\Delta c c o N$ did not regain the ability to grow under these conditions, even after prolonged incubation. Like other $c b b_{3}$ mutants of $R$. sphaeroides ( $\mathrm{O}^{\prime}$ Gara et al., 1998), $\Delta c c o N$ had elevated pigmentation under oxic conditions (data not shown).

As expected, nirK was not expressed in $\Delta c c o N$ cells grown under non-photosynthetic conditions in sealed flasks that were made micro-oxic by the respiratory activity of the growing cells. Under these conditions the cells lacked detectable Nir activity (Fig. 1), nitrite levels of $>3 \mathrm{mM}$ were observed, and the nitrite was not consumed even after incubation for up to $72 \mathrm{~h}$ (data not shown). However, when $\Delta c c o N$ was grown under photosynthetic conditions in sealed vials that were made micro-oxic via cell respiration, Nir activity was detected during active growth and no nitrite accumulated in the medium (Fig. 1). Cells from these cultures did not regain the ability to grow under low-oxygen conditions. Wrapping the vials in foil to prevent photosynthetic growth restored the Nir-deficient phenotype of $\Delta c c o N$.

Besides the difference in light conditions, photosynthetic cultures were also grown at slower agitation rates than cultures growing under non-photosynthetic conditions. To test if the restoration of Nir activity was influenced by agitation rate, cells were grown under photo-denitrifying conditions at the faster agitation rate of the dark experiments. Under these conditions $\Delta c c o N$ had no Nir activity (Fig. 1) and nitrite accumulated to high levels. These results show that the Nir-deficient phenotype is conditional. Manipulation of culture conditions can restore nirK expression and Nir activity, indicating that $c b b_{3}$ activity or expression is not obligatory for nirK expression.

\section{Impact of constitutive nirK expression in a $\Delta c c 0 N$ background}

The simplest interpretation of the phenotype caused by inactivation of $c c o N$ is that the loss of $c b b_{3}$ affects the function of one of the regulatory proteins required for nirK expression. However, since Nir activity is required for nirK expression (Tosques et al., 1997) it cannot be excluded that it is Nir assembly or activity that is being affected in $\Delta c c o N$. To test this possibility, the plasmid pAKHNIR, which contains nirK under control of a constitutive promoter, was conjugated into $\Delta c c o N$. This expression system has been used previously to demonstrate that the loss of Nir activity in a mutant of $R$. sphaeroides 2.4 .3 lacking both cytochromes $c_{2}$ and $c_{\mathrm{Y}}$ was due to a change in nirK expression, not to the loss of electron donors to Nir (Laratta et al., 2006). Unexpectedly, $\Delta c c o N$ with pAKHNIR

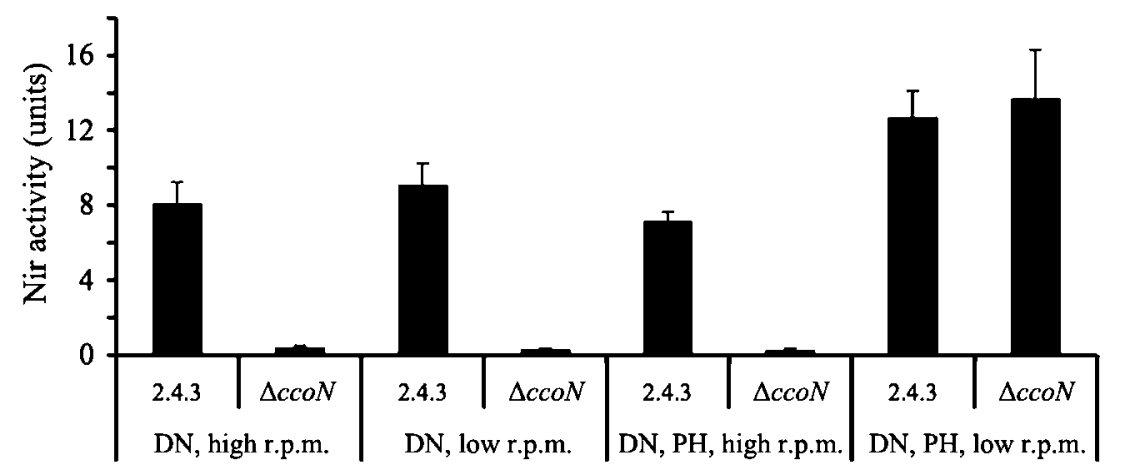

Fig. 1. Nir activity of 2.4 .3 and $\Delta c c o N$ under denitrifying (DN) and denitrifying-photosynthetic (DN, PH) conditions. Under each condition, cultures were grown for at least $48 \mathrm{~h}$ at two different mixing rates; high (250 r.p.m.) and low (50 r.p.m). 
showed very slow growth in nitrate-supplemented medium, even under oxic conditions (Fig. 2). The slow growth of the $\Delta c c o N$ strain was nitrate dependent since growth was not slowed in medium lacking nitrate (Fig. 2). Nir activity could be detected in cells of the $\Delta c c o N$ strain grown in medium without nitrate. Using a methyl-viologendependent assay Nir activity was 10 -fold higher in $\Delta c c o N$ with pAKHNIR than without (Fig. 3).

pAKHNIR was mobilized into several other strains of $R$. sphaeroides 2.4.3 to determine if any had the nitratedependent growth inhibition shown by $\Delta c c o N$. Strains lacking either Nor or NnrR showed no significant growth inhibition in nitrate-supplemented medium until cultures reached optical densities consistent with the onset of denitrification (data not shown). Once these cultures reached optical densities where denitrification genes are typically induced, growth stopped. A strain in which $c y c A$, which encodes cytochrome $c_{2}$, an important electron donor to $c b b_{3}$, was deleted also showed no growth inhibition in the presence of nitrate (Daldal et al., 2001).

\section{Influence of $\mathrm{O}_{2}$ on the growth of $\Delta \mathrm{ccoN}$}

The slow growth of $\Delta c c o N$ with pAKHNIR in the presence of nitrate is consistent with Nir activity producing NO, which inhibits respiration. This suggests that the loss of the $\mathrm{Cbb}_{3}$ oxidase does not prevent Nir assembly or function. Therefore, the inhibition of nirK expression in $\Delta c c o N$ is most probably due to an impact on a regulatory factor. PrrA is known to be required for nirK expression but the increase in PrrA-P levels seen in a $c b b_{3}$ mutant would seem unlikely to inhibit nirK expression (Laratta et al., 2002).

NnrR is the other known regulator of nirK expression. Current evidence suggests NnrR activity is controlled by NO (Kwiatkowski \& Shapleigh, 1996). Members of the $c b b_{3}$ family have been shown to have NO reductase activity (Forte et al., 2001) so loss of this oxidase might lead to an increase in NO levels. Previous work has shown that the loss of Nor also leads to accumulation of NO but this does

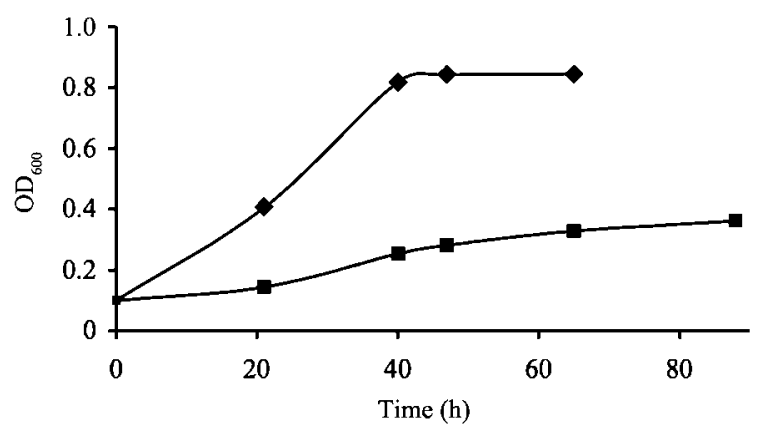

Fig. 2. Growth of $\Delta c c o N$ with a plasmid (pAKHNIR) that constitutively expresses nirK. Cultures were grown in the dark in stoppered flasks with $10 \mathrm{mM}$ nitrate $(\boldsymbol{\square})$ or without nitrate $(\boldsymbol{\nabla})$. Optical density at $600 \mathrm{~nm}$ was monitored over time.

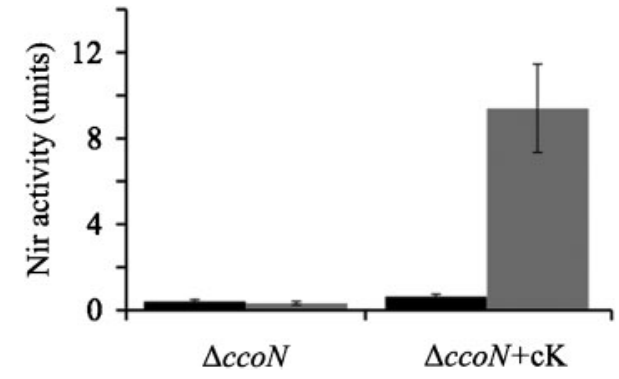

Fig. 3. Nir activity of $\Delta c c o N$ and $\Delta c c o N$ carrying a plasmid (pAKHNIR) that constitutively expresses nirK (cK). Cultures were grown for $24 \mathrm{~h}$ in the dark in stoppered flasks with $10 \mathrm{mM}$ nitrate. Black bars represent whole-cell Nir activity, which measures endogenous electron flow to Nir. Grey bars represent Nir activity using the artificial electron donor methyl viologen as an overall indicator of Nir presence and functionality.

not inhibit NnrR function since nirK and nor are still expressed in a strain lacking Nor activity (Bartnikas et al., 1997; Kwiatkowski \& Shapleigh, 1996). Therefore, it seems unlikely that changes in NO levels are responsible for the observed changes in nirK expression.

Previous work using a heterologous expression system has suggested that a member of the DNR/Nnr family is $\mathrm{O}_{2}$ sensitive (Lee et al., 2006). nirK expression is most inhibited in $\triangle c c o N$ in sealed vessel experiments in which $\mathrm{O}_{2}$ levels are reduced by cellular respiration. Under these conditions, the loss of $c b b_{3}$ will affect the end-point level of $\mathrm{O}_{2}$ in the cultures. The two major respiratory oxidases in $R$. sphaeroides 2.4.3 have different affinities for oxygen. The $c b b_{3}$ oxidases have been shown to have a $K_{\mathrm{m}}$ for $\mathrm{O}_{2}$ that is in the low nanomolar range while the $K_{\mathrm{m}}$ of the $a a_{3}$ oxidase is at the low micromolar level (Preisig et al., 1996; Riistama et al., 2000). Loss of the $c b b_{3}$ oxidase will thus result in a higher end-point $\mathrm{O}_{2}$ level inside the sealed incubations used in this work. Therefore, it is not unreasonable to suggest that the lack of nirK expression in $\Delta c c o N$ could be due to the inhibition of NnrR by $\mathrm{O}_{2}$. To test if this residual $\mathrm{O}_{2}$ is responsible for inhibition of nirK expression a series of experiments was carried out in sealed vials with a range of headspace oxygen concentrations established using gas mixes (Fig. 4). As expected, the wild-type strain 2.4.3 grew in the dark under $\mathrm{O}_{2}$ concentrations ranging from atmospheric $(20 \%)$ down to the sample with no added $\mathrm{O}_{2}$. Even at the lowest $\mathrm{O}_{2}$ concentrations growth was not nitrate dependent. Nevertheless, wild-type cells began to denitrify at approximately $2 \%$ oxygen and below, as indicated by the onset of Nir activity. In agreement with these results, nirK expression could be detected in cultures grown with $\leqslant 1 \% \mathrm{O}_{2}$ (data not shown).

$\Delta c c o N$ grew in vials with oxygen levels ranging from atmospheric to $2 \%$ and this growth was not nitrate dependent. Unlike the wild-type strain, however, no growth was observed in the vials for incubations up to 10 days if $\mathrm{O}_{2}$ levels were $<2 \%$ or $>0.2 \%$, irrespective of the presence of 


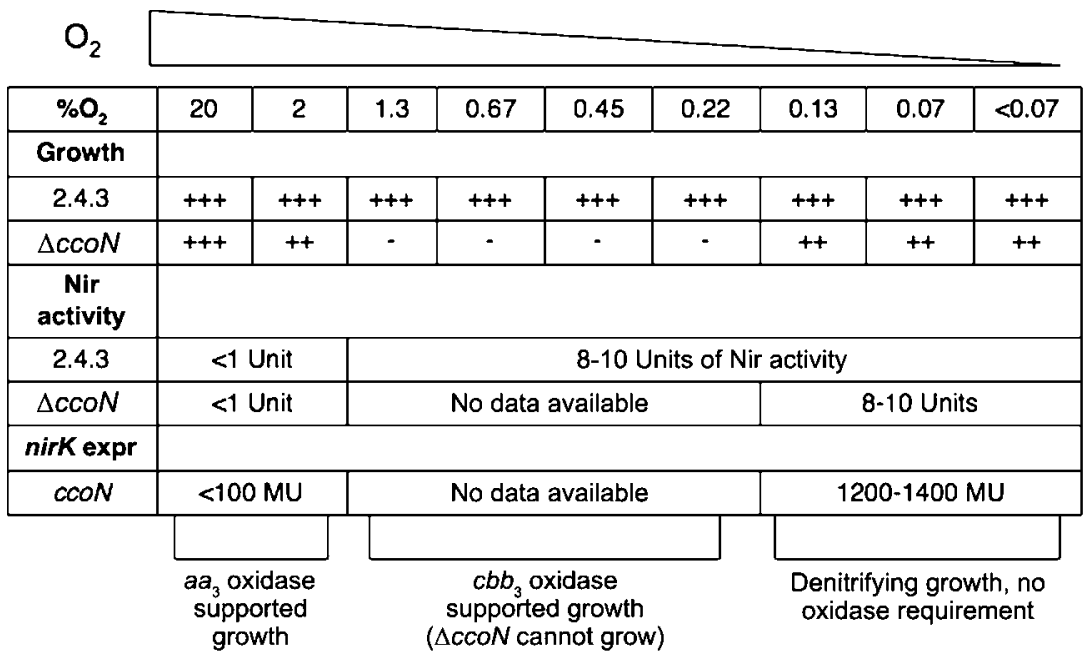

Fig. 4. Growth of 2.4 .3 and $\Delta c c o N$ under a range of established oxygen concentrations. Oxygen concentration is represented as a percentage, with atmospheric oxygen considered $20 \%$. For each oxygen concentration, growth, Nir activity and nirK-lacZ expression were assessed. Expression values are in Miller units (MU). nitrate. Growth was observed with $\mathrm{O}_{2}$ levels $<0.2 \%$ after 5 days but only if nitrate was present, demonstrating that growth was due to denitrification. Cells of the $c c o N$ mutant grown under these low $\mathrm{O}_{2}$ conditions had wild-type levels of Nir activity (Fig. 4) and nirK and nor expression. One possible explanation for the lack of growth under intermediate $\mathrm{O}_{2}$ conditions is that there is no oxidase expressed due to the absence of the $c b b_{3}$ gene and a decrease in expression of the genes encoding the $a a_{3}$ oxidase (Pappas et al., 2004). However, cells of $\Delta c c o N$ grown at $0.07 \% \quad \mathrm{O}_{2}$ on nitrate-containing medium consumed $\mathrm{O}_{2}$ at the same rate as the wild-type strain grown under identical conditions.

To further test the role of $\mathrm{O}_{2}$ in controlling the denitrifying ability of $\Delta c c o N$, a co-culture experiment was carried out. Growing $\Delta c c o N$ in a sealed flask together with a strain containing an oxidase with an $\mathrm{O}_{2}$ affinity equivalent to that of the $c b b_{3}$ in strain 2.4.3 should restore nirK expression and $\mathrm{Nir}$ activity in $\Delta c c o N$ if end-point $\mathrm{O}_{2}$ levels are critical. Conveniently, the $R$. sphaeroides type strain 2.4.1 has an aerobic respiratory chain identical to 2.4.3 but lacks nirK (Kwiatkowski et al., 1997). Co-cultures with either a $1: 3$ or $3: 1$ ratio of wild-type 2.4 .3 or $\Delta c c o N$ to 2.4.1 cells were used for these experiments. Initial experiments done in the dark indicated that 2.4.1 outcompeted $\Delta c c o N$ by at least $30: 1$ based on c.f.u. $\mathrm{ml}^{-1}$ values determined at the end of the experiment (data not shown). The poor growth of $\Delta c c o N$ under these conditions is consistent with the inability of $\Delta c c o N$ to efficiently respire at low $\mathrm{O}_{2}$ concentrations. To overcome this problem the co-culture experiments were carried out under high-agitation photosynthetic conditions, which were shown to prevent nirK expression in $\Delta c c o N$ (Fig. 1). When cultures were grown under these conditions the ratios of $\Delta c c o N$ to 2.4 .1 did not change significantly during the course of the experiment. As expected, no nitrite accumulated in the co-cultures and the cells showed near wild-type levels of Nir activity (Fig. 5).

\section{Mutant isolation}

Attempts were made to isolate mutants of $\Delta c \operatorname{coN}$ that could grow via nitrate-dependent respiration at $\mathrm{O}_{2}$ levels between 0.2 and $2.0 \%$. Both spontaneous and UV-induced mutagenesis were used in an effort to generate mutants. Various selective conditions were tried, including photosynthetic conditions, low-oxygen conditions without nitrate and transitions from no-nitrate to nitrate-containing medium. After repeated attempts, involving incubation times of up to 3 months, no strains of $\Delta c c o N$ were isolated that would grow in nitrate-containing medium at these $\mathrm{O}_{2}$ levels.

\section{DISCUSSION}

nirK expression requires the direct or indirect involvement of multiple regulators that integrate information about environmental conditions (Laratta et al., 2002; Tosques et al., 1997). In strain 2.4.3, nitrate is the initial substrate for denitrification, making all downstream denitrification

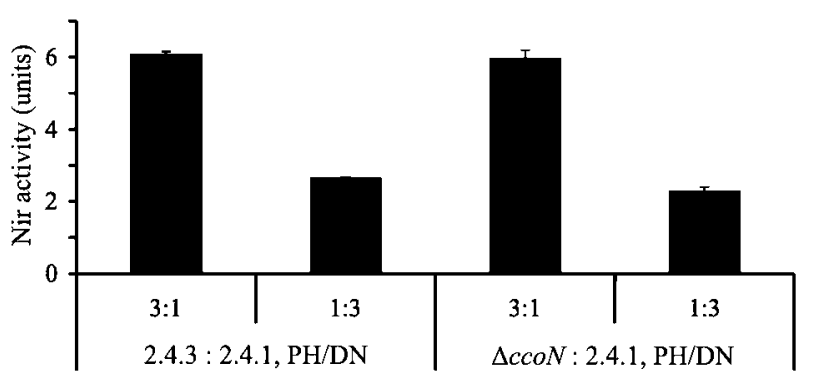

Fig. 5. Nir activity of co-cultures of strains 2.4 .3 or $\Delta c c o N$ with strain 2.4.1. In all cases cultures were grown for $36 \mathrm{~h}$ under photosynthetic-denitrifying conditions. Co-cultures were started in two different ratios, $3: 1$ and $1: 3$, which represent the starting quantity of $2.4 .3: 2.4 .1$ or $\Delta c c o N: 2.4 .1$. 
regulation dependent on the expression of nitrate reductase. Nitrate reductase is upregulated by an unknown mechanism in response to decreasing oxygen levels (A. Hartsock \& J. P. Shapleigh, unpublished). The nitrite produced by nitrate reduction is then reduced to NO by the low levels of Nir produced by basal levels of nirK expression. NO then activates DNA binding by NnrR, which is necessary but not sufficient for expression of nirK (Kwiatkowski \& Shapleigh, 1996; Tosques et al., 1996, 1997). nirK expression also requires activation by PrrA-P (Laratta et al., 2002). Production of PrrA-P occurs when $\mathrm{O}_{2}$ levels are low enough to limit turnover of the $c b b_{3}$ oxidase (Oh et al., 2004). A decrease in $\mathrm{O}_{2}$ is not required to produce high levels of PrrA-P in cells lacking the $c b b_{3}$ oxidase (O'Gara et al., 1998). If there are no additional layers of regulation involved it would be predicted that nirK expression should occur at higher $\mathrm{O}_{2}$ levels in a $c c o N$ mutant than in the wild-type. The contradictory observation that the loss of the $c b b_{3}$ oxidase reduced nirK expression suggested there are additional uncharacterized factors required for nirK expression, which motivated this study.

The experiments described here suggest that the conditional nirK expression phenotype in $\Delta c c o N$ is a consequence of the loss of a high-affinity oxidase which, under certain growth conditions, leaves $\mathrm{O}_{2}$ levels so high as to prevent nirK expression. The restoration of Nir activity in $\Delta c c o N$ cells grown in vials whose atmosphere has been sparged with a low- $\mathrm{O}_{2}$ gas shows that nirK expression can be readily restored in this strain and that $c b b_{3}$ activity is not obligatory for nirK expression. $c b b_{3}$ is only required for nirK expression when aerobic respiration is being used to lower $\mathrm{O}_{2}$ concentrations during growth.

The likely oxygen-sensitive component that is being affected by the loss of $c b b_{3}$ is NnrR. The loss of the $c b b_{3}$ oxidase does not affect photosynthetic growth (O'Gara et al., 1998), suggesting that a factor specifically associated with denitrification is being affected. Since nitrate reductase is functional in $\Delta c c o N$, this indicates that the factor being affected is either Nir or Nor specifically or some factor involved in regulation of nirK as well as nor. The activities of Nir and Nor are not $\mathrm{O}_{2}$ sensitive, suggesting it is the regulation of their expression that is affected (Morley et al., 2008). Since PrrA is functional in $\Delta c c o N$, this leaves $\mathrm{NnrR}$ as the probable $\mathrm{O}_{2}$-sensitive component. This conclusion is supported by previous work which suggested that the regulator of the genes encoding the NO-forming nitrite reductase and NO reductase in Paracoccus denitrificans, which, like NnrR, is also a member of the FNR family, is $\mathrm{O}_{2}$ sensitive (Lee et al., 2006). Having regulators of this type be sensitive to $\mathrm{O}_{2}$ ensures that $\mathrm{NO}$ production does not occur until $\mathrm{O}_{2}$ levels are extremely low. This reduces the possibility of the formation of reactive nitrogen oxides that can arise due to the interaction of $\mathrm{NO}$ with various oxygen species (Hughes, 2008; Patel et al., 1999). This layer of $\mathrm{O}_{2}$-dependent regulation also ensures that denitrification is secondary to $\mathrm{O}_{2}$-dependent regulation at a wide range of $\mathrm{O}_{2}$ levels. However, while $\mathrm{O}_{2}$ concentrations must be low for nirK expression it is notable that it can be expressed at $\mathrm{O}_{2}$ levels that are high enough to support aerobic respiration via the $c b b_{3}$ oxidase (Fig. 4). This makes denitrification a micro-oxic process and suggests cells can co-respire oxygen and nitrate.

While $c b b_{3}$ oxidase activity was needed for nirK expression if cell respiration was being used to reduce $\mathrm{O}_{2}$ levels in cultures growing in the dark, this was not always the case for cells growing photosynthetically. This may indicate that in addition to being $\mathrm{O}_{2}$ sensitive, the activation of $\mathrm{NnrR}$ function also requires ATP synthesis. In cultures growing in the dark, once $\mathrm{O}_{2}$ levels reach concentrations where the $a a_{3}$ oxidase is no longer active there is no longer rapid ATP synthesis due to the absence of the $c b b_{3}$ oxidase. This is evidenced by the overgrowth of strain 2.4.1 in the co-culture experiments. Cells grown in the light can generate ATP via photosynthesis after $\mathrm{O}_{2}$ levels are reduced. A lack of ATP synthesis alone, however, cannot account for the conditional nirK expression phenotype in $\Delta c c o N$. The importance of $\mathrm{O}_{2}$ sensitivity is demonstrated by the observation that there is no expression of nirK in $\Delta c c o N$ at $\mathrm{O}_{2}$ levels where $a a_{3}$ is functional. The impact of increased agitation on nirK expression in cells growing photosynthetically also suggests $\mathrm{O}_{2}$ plays a role in inhibiting nirK expression under these conditions. Rapid agitation would vigorously mix $\mathrm{O}_{2}$ throughout the culture medium. Slower agitation and the consequent poorer mixing of $\mathrm{O}_{2}$ may provide conditions where NnrR is functional and the ATP also necessary for its assembly or activation is available from photosynthesis. Another possibility is that the light used to allow photosynthetic growth is causing photolysis of an NO adduct that would otherwise be preventing oxygen consumption.

An unexpected observation made during these experiments was the nitrate sensitivity of the $\Delta c c o N$ strain containing pAKHNIR. Since this phenotype requires both the constitutively expressed nirK and the presence of nitrate in the medium, it is likely that it is due to the constitutively expressed Nir producing NO, which inhibits respiration. This was unexpected because a wild-type strain with the same nirK expression plasmid is not nitrate sensitive and also because strains lacking Nor activity can grow in the presence of nitrate at high $\mathrm{O}_{2}$ levels (Bartnikas et al., 1997; Tosques et al., 1996). Therefore, the only strain that is affected at high $\mathrm{O}_{2}$ concentrations is the one lacking $c b b_{3}$ activity with pAKHNIR. One possible explanation for this is that the $c b b_{3}$ oxidase is NO resistant or is capable of mitigating NO toxicity. The latter possibility is consistent with the observation that some $c b b_{3}$ oxidases have NO reductase activity (Forte et al., 2001). While the $c b b_{3}$ in strain 2.4.3 may have some NO reductase activity, it is not capable of reducing $\mathrm{NO}$ at rates fast enough to keep up with NO production by Nir during denitrification (Bartnikas et al., 1997). Strains in which nor has been disrupted stop growing when Nir becomes active even if $c b b_{3}$ is present, demonstrating the importance of Nor 
under denitrification conditions (Bartnikas et al., 1997). It is also possible that an aerobic mechanism for mitigating $\mathrm{NO}$ toxicity is disrupted in $\Delta c c o N$, given the global nature of the PrrBA regulon (Eraso et al., 2008).

Experiments described here and in previous publications indicate that oxygen control of nirK expression, and consequently denitrification, in $R$. sphaeroides 2.4 .3 is achieved through both PrrBA and NnrR. The activity of these two regulatory systems is directly or indirectly influenced by the $c b b_{3}$ oxidase. This suggests a general design feature in denitrifiers whereby the $\mathrm{O}_{2}$ level at which the expression of genes encoding products involved in $\mathrm{NO}$ production and reduction is adjusted to correlate with the $K_{\mathrm{m}}$ of the highest affinity $\mathrm{O}_{2}$-reducing oxidase in the respiratory chain. If true aerobic denitrification does occur, it may require a bacterium lacking high-affinity oxidases.

\section{ACKNOWLEDGEMENTS}

We wish to thank Sam Kaplan and Jeong-Il Oh for the generous gifts of plasmids and strains. This work was supported by the US Department of Energy (95ER20206).

\section{REFERENCES}

Baek, S. H., Hartsock, A. \& Shapleigh, J. P. (2008). Agrobacterium tumefaciens C58 uses ActR and FnrN to control nirK and nor expression. J Bacteriol 190, 78-86.

Bartnikas, T. B., Tosques, I. E., Laratta, W. P., Shi, J. \& Shapleigh, J. P. (1997). Characterization of the nitric oxide reductase-encoding region in Rhodobacter sphaeroides 2.4.3. J Bacteriol 179, 35343540.

Bedmar, E. J., Robles, E. F. \& Delgado, M. J. (2005). The complete denitrification pathway of the symbiotic, nitrogen-fixing bacterium Bradyrhizobium japonicum. Biochem Soc Trans 33, 141-144.

Chung, C. T., Niemela, S. L. \& Miller, R. H. (1989). One-step preparation of competent Escherichia coli: transformation and storage of bacterial cells in the same solution. Proc Natl Acad Sci U S A 86, 2172-2175.

Daldal, F., Mandaci, S., Winterstein, C., Myllykallio, H., Duyck, K. \& Zannoni, D. (2001). Mobile cytochrome $c_{2}$ and membrane-anchored cytochrome $c_{\mathrm{Y}}$ are both efficient electron donors to the $c b b_{3}$ - and $a a_{3^{-}}$ type cytochrome $c$ oxidases during respiratory growth of Rhodobacter sphaeroides. J Bacteriol 183, 2013-2024.

de Bruijn, F. J., Rossbach, S., Bruand, C. \& Parrish, J. R. (2006). A highly conserved Sinorhizobium meliloti operon is induced microaerobically via the FixLJ system and by nitric oxide (NO) via NnrR. Environ Microbiol 8, 1371-1381.

Eraso, J. M., Roh, J. H., Zeng, X., Callister, S. J., Lipton, M. S. \& Kaplan, S. (2008). Role of the global transcriptional regulator PrrA in Rhodobacter sphaeroides 2.4.1: combined transcriptome and proteome analysis. J Bacteriol 190, 4831-4848.

Forte, E., Urbani, A., Saraste, M., Sarti, P., Brunori, M. \& Giuffrè, A. (2001). The cytochrome $c b b_{3}$ from Pseudomonas stutzeri displays nitric oxide reductase activity. Eur J Biochem 268, 6486-6491.

Giardina, G., Rinaldo, S., Johnson, K. A., Di Matteo, A., Brunori, M. \& Cutruzzola, F. (2008). NO sensing in Pseudomonas aeruginosa: structure of the transcriptional regulator DNR. J Mol Biol 378, $1002-1015$.
Hartsock, A. \& Shapleigh, J. P. (2010). Identification, functional studies, and genomic comparisons of new members of the NnrR regulon in Rhodobacter sphaeroides. J Bacteriol 192, 903-911.

Hughes, M. N. (2008). Chemistry of nitric oxide and related species. Methods Enzymol 436, 3-19.

Kovach, M. E., Elzer, P. H., Hill, D. S., Robertson, G. T., Farris, M. A., Roop, R. M., II \& Peterson, K. M. (1995). Four new derivatives of the broad-host-range cloning vector pBBR1MCS, carrying different antibiotic-resistance cassettes. Gene 166, 175-176.

Kwiatkowski, A. V. \& Shapleigh, J. P. (1996). Requirement of nitric oxide for induction of genes whose products are involved in nitric oxide metabolism in Rhodobacter sphaeroides 2.4.3. J Biol Chem 271, 24382-24388.

Kwiatkowski, A. V., Laratta, W. P., Toffanin, A. \& Shapleigh, J. P. (1997). Analysis of the role of the $n n r R$ gene product in the response of Rhodobacter sphaeroides 2.4 .1 to exogenous nitric oxide. J Bacteriol 179, 5618-5620.

Laratta, W. P., Choi, P. S., Tosques, I. E. \& Shapleigh, J. P. (2002). Involvement of the PrrB/Pra two-component system in nitrite respiration in Rhodobacter sphaeroides 2.4.3: evidence for transcriptional regulation. J Bacteriol 184, 3521-3529.

Laratta, W. P., Nanaszko, M. J. \& Shapleigh, J. P. (2006). Electron transfer to nitrite reductase of Rhodobacter sphaeroides 2.4.3: examination of cytochromes $c_{2}$ and $c_{\mathrm{Y}}$. Microbiology 152, 1479-1488.

Lee, Y. Y., Shearer, N. \& Spiro, S. (2006). Transcription factor NNR from Paracoccus denitrificans is a sensor of both nitric oxide and oxygen: isolation of $n n r^{\star}$ alleles encoding effector-independent proteins and evidence for a haem-based sensing mechanism. Microbiology 152, 1461-1470.

Lueking, D. R., Fraley, R. T. \& Kaplan, S. (1978). Intracytoplasmic membrane synthesis in synchronous cell populations of Rhodopseudomonas sphaeroides. J Biol Chem 253, 451-457.

Maniatis, T., Fritsch, E. F. \& Sambrook, J. (1982). Molecular Cloning: a Laboratory Manual. Cold Spring Harbor, NY: Cold Spring Harbor Laboratory.

Morley, N., Baggs, E. M., Dorsch, P. \& Bakken, L. (2008). Production of $\mathrm{NO}, \mathrm{N}_{2} \mathrm{O}$ and $\mathrm{N}_{2}$ by extracted soil bacteria, regulation by $\mathrm{NO}_{2}$ and $\mathrm{O}_{2}$ concentrations. FEMS Microbiol Ecol 65, 102-112.

O’Gara, J. P., Eraso, J. M. \& Kaplan, S. (1998). A redox-responsive pathway for aerobic regulation of photosynthesis gene expression in Rhodobacter sphaeroides 2.4.1. J Bacteriol 180, 4044-4050.

Oh, J. I. (2006). Effect of mutations of five conserved histidine residues in the catalytic subunit of the $c b b_{3}$ cytochrome $c$ oxidase on its function. J Microbiol 44, 284-292.

Oh, J. I., Ko, I. J. \& Kaplan, S. (2004). Reconstitution of the Rhodobacter sphaeroides $c_{b} b_{3}$-PrrBA signal transduction pathway in vitro. Biochemistry 43, 7915-7923.

Pappas, C. T., Sram, J., Moskvin, O. V., Ivanov, P. S., Mackenzie, R. C., Choudhary, M., Land, M. L., Larimer, F. W., Kaplan, S. \& Gomelsky, M. (2004). Construction and validation of the Rhodobacter sphaeroides 2.4.1 DNA microarray: transcriptome flexibility at diverse growth modes. J Bacteriol 186, 4748-4758.

Patel, R. P., McAndrew, J., Sellak, H., White, C. R., Jo, H., Freeman, B. A. \& Darley-Usmar, V. (1999). Biological aspects of reactive nitrogen species. Biochim Biophys Acta 1411, 385-400.

Preisig, O., Zufferey, R., Thony-Meyer, L., Appleby, C. A. \& Hennecke, H. (1996). A high-affinity $c b b_{3}$-type cytochrome oxidase terminates the symbiosis-specific respiratory chain of Bradyrhizobium japonicum. J Bacteriol 178, 1532-1538.

Prentki, P. \& Krisch, H. M. (1984). In vitro insertional mutagenesis with a selectable DNA fragment. Gene 29, 303-313. 
Ranson-Olson, B. \& Zeilstra-Ryalls, J. (2008). Regulation of the Rhodobacter sphaeroides 2.4.1 hemA gene by PrrA and FnrL. J Bacteriol 190, 6769-6778.

Riistama, S., Puustinen, A., Verkhovsky, M. I., Morgan, J. E. \& Wikstrom, M. (2000). Binding of $\mathrm{O}_{2}$ and its reduction are both retarded by replacement of valine 279 by isoleucine in cytochrome c oxidase from Paracoccus denitrificans. Biochemistry 39, 63656372.

Risgaard-Petersen, N., Langezaal, A. M., Ingvardsen, S., Schmid, M. C., Jetten, M. S., Op, C., Derksen, J. W., Pina-Ochoa, E., Eriksson, S. P. \& other authors (2006). Evidence for complete denitrification in a benthic foraminifer. Nature 443, 93-96.

Simon, R.,Priefer, U. \& Pühler, A. (1983). A broad host range mobilization system for in vitro genetic engineering: transposon mutagenesis in gram negative bacteria. Nature Biotechnology 1, 784791.
Spiro, S. (2007). Regulators of bacterial responses to nitric oxide. FEMS Microbiol Rev 31, 193-211.

Tosques, I. E., Shi, J. \& Shapleigh, J. P. (1996). Cloning and characterization of $n n r R$, whose product is required for the expression of proteins involved in nitric oxide metabolism in Rhodobacter sphaeroides 2.4.3. J Bacteriol 178, 4958-4964.

Tosques, I. E., Kwiatkowski, A. V., Shi, J. \& Shapleigh, J. P. (1997). Characterization and regulation of the gene encoding nitrite reductase in Rhodobacter sphaeroides 2.4.3. J Bacteriol 179, 1090-1095.

Yanisch-Perron, C., Vieira, J. \& Messing, J. (1985). Improved M13 phage cloning vectors and host strains: nucleotide sequences of the M13mp18 and pUC19 vectors. Gene 33, 103-119.

Zumft, W. G. (1997). Cell biology and molecular basis of denitrification. Microbiol Mol Biol Rev 61, 533-616.

Edited by: J. Moir 